\title{
Un primer análisis de las relaciones contractuales que se establecen en la actividad de intermediación turística cubana
}

\section{A first analysis of the contractual relations established in the Cuban tourist brokerage activity}

\author{
*Martínez Montenegro, I. ${ }^{1}$; Baeza Leiva, M. ${ }^{1}$
}

${ }^{1}$ Facultad de Ciencias Jurídicas en la Universidad Católica de Temuco. Chile

\section{RESUMEN}

En la presente investigación se abordaron los fundamentos jurídicos que deben regir en las relaciones contractuales que se establecen en la actividad de intermediación turística en Cuba $y$, en especial, a la figura del contrato de viajes, para lograr el eficaz funcionamiento de estas en Cuba, en el marco de una nueva realidad social y económica que se presenta. A tales efectos, se determinaron las bases jurídicas que deben sustentar las relaciones contractuales para contribuir al perfeccionamiento de su régimen jurídico. Acorde con los resultados obtenidos, con este estudio teórico-doctrinal se alcanza la armonía que demanda la política turística interna, para lograr un mayor y más eficaz, respaldo legal en su funcionamiento.

Palabras clave: Derecho del turismo; contrato de viaje; intermediación turística.

\section{ABSTRACT}

In this research the legal basis to govern the contractual relations established in the activity of tourism intermediation in Cuba and especially the figure of the travel contract addressed to achieve the effective functioning of the same in Cuba, in the framework of a new social and economic reality presented. To this end, the legal bases that should underpin contractual relations to contribute to the improvement of their legal status determined. According to the results obtained with this study theoretical and doctrinal harmony that demands internal tourism policy to achieve greater and more effective legal, backup operation is reached.

KEYWORDS: Right tourism; travel contract; tourist intermediation.

\section{INTRODUCCIÓN}

La categoría jurídica de mediación o intermediación turística es un tipo especial de operación mercantil que se desarrolla a través de la puesta en contacto del productor y el consumidor de un producto por medio de personas cuya prestación contractual consiste en la promoción o concertación de operaciones comerciales. Nacen como resultado del desarrollo económico del siglo XIX y la ampliación de los mercados, las mejoras en los medios de comunicación y de transporte, las necesidades derivadas de la descentralización productiva, de la evolución tecnológica y los nuevos sistemas de producción; así como del progreso de diversas relaciones jurídicas ya existentes y dotadas de su

\footnotetext{
*Autor Correspondiente: Isnel Martínez Montenegro. Facultad de Ciencias Jurídicas de la Universidad Católica de Temuco. Chile.

E-mail: isnel.martinez1986@gmail.com

Fecha de recepción: setiembre 2016; Fecha de aceptación: octubre 2016
} 
propio régimen jurídico, a partir de las cuales se produjo la tipificación legal de nuevas actividades de mediación o intermediación en el tráfico mercantil (Cairós, 2001, p.121).

Un papel fundamental es el rol que desempeñan las empresas de intermediación turística en la unificación de los diferentes factores que conforman la red de comercialización del producto turístico, debido en gran medida, a que son entidades que organizan servicios turísticos diversos (viajes combinados, excursiones, representación de otras agencias de viajes (AA.VV.) y a que facilitan, a nivel mundial y nacional, su mercantilización de conjunto con los servicios ofrecidos por otras empresas (fundamentalmente reservas de plazas en medios de transporte de viajeros y de alojamientos turísticos), que sirve de enlace principal, en muchas ocasiones, y necesariamente imprescindibles entre la oferta y la demanda turística.

Al respecto, es necesario apuntar que en el ámbito internacional varios autores han tratado el régimen jurídico de las empresas de intermediación turística, motivados fundamentalmente por el interés de la doctrina mercantilista en el estudio de la legislación de viajes combinados, la responsabilidad de las AA.VV., los contratos mercantiles en el campo del turismo, la intervención administrativa, entre otros. Desde esta diferente perspectiva se destacan en las últimas décadas a los autores García Rubio, Gómez Calero, Martínez Espín, Sanz Domínguez, Kemelmajer de Carlucci de, Bech Serrat (Gómez Calero, 1975, García Rubio, 1999; Martínez Espín, 1999; Sanz Domínguez, 20015; Kemelmajer de Carlucci, 2006, Bech Serrat, 2007).

En el entorno nacional existen diversos autores que desde el punto de vista económico, turístico y directivo, han tratado la problemática. Desde estos diversos enfoques científicos se destacan en los últimos años Guevara Hernández, Jiménez Valero y Suárez Mella (Guevara Hernádez, 2009; Jiménez Valero y Suárez Mella, 2013; Martínez Montenegro 2014).

En cambio, desde el punto de vista jurídico, existe una marcada carencia de obras científicas dedicadas a la actividad turística de intermediación y el estudio de su régimen jurídico, lo que nos pone en conexión, básicamente, con el mundo de las AA.VV., cada vez más nutrido y diverso, y ante una realidad social y económica de dimensiones extraordinarias como nos muestran algunos datos de campo contenidos en la tesis. En el ámbito del turismo y sus variadas formas o tipos de servicios, la intermediación turística es la actividad que hace posible el desarrollo de las demás.

La infinidad de relaciones jurídicas que esta intermediación genera y, por consecuencia, la envergadura alcanzada por el sector ya justificaría la importancia de un estudio de este tipo (Martínez Montenegro, 2012); pero la razón verdadera de la elección del tema, no estriba tan solo en ese factor cuantitativo, pues si tal fuera este el dato determinante, la misma razón hubiera concurrido para estudiar los fundamentos de estos mediadores en cualquier otra clase de servicios turísticos (hostelería, restauración, alojamientos rurales), y hasta en otros sectores de la economía, tan en auge, como el que es objeto de esta investigación.

\section{Análisis de las diferentes modalidades contractuales presentes en el funcionamiento de las AA.VV. cubanas.}

Las AA.VV. cubanas desarrollan la actividad turística que ofertan a sus clientes a través de las relaciones económicas que establecen con los prestatarios de los servicios turísticos. Para ello, como cualquier empresa, unidad presupuestada o forma de gestión no estatal legalizan sus relaciones 
entre sí, mediante contratos de acurdo con las disposiciones y los tipos de contratos regulados en el Decreto Ley 304 de 2012 de la contratación económica, el Decreto 310 de los tipos de contratos económicos y las disposiciones de Código Civil cubano (Decreto, 2012).

En la cadena contractual que se produce la A.V. se obliga, en su propio nombre, a prestar un conjunto combinado de servicios, por lo menos dos: transporte $\mathrm{y}$ hospedaje $\mathrm{u}$ otro servicio turístico que constituya una parte significativa del viaje ${ }^{1}$. Estos contratos, si bien aparecen como independientes, presentan una estrecha vinculación entre sí, pues todos responden a un mismo resultado económico: la realización de un viaje a cambio de un precio global.

La regulación del contrato de transporte se establece a partir del artículo 116 al 149 del Decreto 310 de 2012, en que se estipulan las clasificaciones del mismo de acuerdo a la vía utilizada para efectuar la transportación, por vía terrestre que incluye el transporte ferroviario; por vías marítimas, fluviales, o lacustres y por vías aéreas; además a los contratos de transporte también les son aplicables las normas de carácter supletorio establecidas en la legislación especial vigente y los tratados o convenios internacionales en los que la República de Cuba es parte según el apartado 117. Las disposiciones generales, el contenido y las obligaciones de cada una de sus modalidades son abordados en las disposiciones del citado Decreto (Decreto, 2012).

En relación con hospedaje, su regulación se halla a partir del artículo 438 al 443 del Código Civil cubano y las normas de carácter supletorio establecidas en la legislación especial vigente y los tratados o convenios internacionales en los que la República de Cuba es parte (Ley, 1987).

De tal forma, las AA.VV. y los prestatarios, basan sus relaciones contractuales de carácter económico en dos figuras en específico: el contrato de transportación y el de hospedaje. También en algunos casos pueden concertar otros servicios turísticos que se constituyen como parte no accesoria de ambos contratos.

En conjunto con los esfuerzos desde el punto de vista legal, cuestiones económicas y de opciones en la oferta de los prestatarios de servicios turísticos, trascienden las posibilidades para lograr impedir un cumplimiento defectuoso de las obligaciones del contrato entre la empresa receptiva y los turistas ${ }^{2}$.

En la actualidad, no existe una diversificación en los servicios y las ofertas complementarias al alojamiento que distingan al país en el sector del turismo, y menos aún que priorice el desarrollo de las modalidades del turismo nacional: turismo de salud, marinas y náutica, golf e inmobiliaria, turismo de aventura y naturaleza, parques temáticos, crucerismo, historia, cultura y patrimonio, convenciones, congresos y ferias, entre otras; para que contribuyan al mejoramiento de la oferta con respecto al precio en el proceso de comercialización. De este modo existen carencias en la calidad y variedad de los servicios que provocan inconformidades, quejas y reclamaciones de los clientes,

\footnotetext{
${ }^{1}$ Pueden ser ejemplos prestaciones no accesorias que formen parte del objeto principal del viaje el aprendizaje de idiomas en el extranjero o presenciar un partido de béisbol.

${ }^{2} U n$ ejemplo es que para ofertar el circuito del oriente no existen en temporada alta opciones suficientes de establecimientos hoteleros en el centro del país para enlazar el recorrido y evitar así que los clientes no se sientan afectados por el cansancio que provoca la distancia del trayecto. También existen supuestos de productos ecológicos en terrenos montañosos donde hay una única empresa de camiones en condiciones de brindar el traslado.
} 
las que se asumen por costos de no calidad. Estos costos no son compensados por los prestatarios, por ausencias de cláusulas en los contratos que regulen este tipo de situaciones en una reclamación del derecho de regreso de la A.V. afectada ${ }^{3}$.

Todo ello conlleva a la imposibilidad de contratar con los proveedores con la anticipación suficiente que permita ofrecer los productos turísticos con los precios vigentes para las temporadas que se contratan y con una mayor eficiencia. Simultáneamente, todas las AA.VV. comercializan los mismos servicios que ofertan, aunque cada una con estrategias de mercado diferentes y por separado, sin embargo, en todas se valen de proformas contractuales preestablecidas donde anexan los productos que brindan, y también, los paquetes turísticos de las que integran su entorno competitivo a partir de la concertación de contratos entre las diferentes AA.VV.

\section{Aspectos relevantes de derecho comparado en el contrato de viajes}

En cuanto a la regulación del contrato de viaje en los países europeos, existe la Directiva núm.90-314 de 1990 que adecua el Convenio de Bruselas del 23 de abril de 1970 relativo al contrato internacional de viajes. En la normativa por su parte, se concibe una definición que para los países de la comunidad se fundamenta en el elemento subjetivo ${ }^{4}$ y el contenido se centra en la regulación del contrato mercantil de viaje combinado, en el programa y la oferta de viajes combinados, la forma y contenido que ha de tener el contrato, la información que habrá de ofrecerse al usuario sobre el viaje combinado, la revisión de precios, modificación del contrato y su resolución, consecuencias de la no prestación de los servicios contratados y el régimen de responsabilidad de los organizadores y detallistas.

Dos supuestos particulares en la regulación del contrato de viajes en Europa, lo constituyen España y Alemania. En el caso de España se promulgó la Ley 21 de 1995 reguladora de los viajes combinados con el objetivo de ordenar esta modalidad del contrato mercantil y de modo especial el régimen de responsabilidad de los organizadores y detallistas.

Por otra parte, la normativa alemana cuenta con una regulación de contrato de viaje en el Código Civil de 1979 que obedece a la necesidad de ofrecer una mayor protección al consumidor de viajes combinados ${ }^{5}$. Contempla distintas medidas específicas que favorecen a los usuarios en la reclamación de indemnización de daños y perjuicios por incumplimiento del contrato por parte de los prestatarios del servicio, tales como de la prohibición de la llamada cláusula de intermediación, el reconocimiento de la acumulación del derecho a la indemnización de daños y perjuicios, junto a otras medidas de defensas del consumidor frente a dicho incumplimiento contractual y al derecho de obtener indemnización por la pérdida de vacaciones programadas.

Por su parte, en Argentina se ratificó la Convención Internacional del Contrato de Viajes de 1972 , que en su artículo $2^{6}$ se establece el campo de aplicación que comprende todo contrato de viaje concluido por un organizador de viajes o por un intermediario de viajes cuando su establecimiento principal o

\footnotetext{
3 Información obtenida de los contratos revisados en el estudio aplicado de la A.V. CUBANACAN Varadero.

${ }^{4}$ Cfr. Art. 2.2 de la Directiva 90-134 de 1992. La persona que organiza de forma no ocasional viajes combinados y los vende $u$ ofrece a la venta, directamente o por medio de un detallista.

${ }^{5}$ Cfr. Artículo 651 del Código Civil Alemán del año 1979.

${ }^{6}$ Vid. Art. 2 de la Convención Internacional sobre Contrato de Viaje de 1970.
} 
a falta del establecimiento, su residencia habitual, o el establecimiento por intermedio del cual el contrato de viaje sea concluido o se encuentre en un Estado contratante. Con respecto a la consideración del contrato de viaje, la Convención Internacional de Bruselas dispone que es aquel por el cual una parte que es la organizadora, se obliga en su propio nombre, mediante un precio global, a prestar un conjunto de servicios combinados de transporte, alojamientos independientes del transporte $u$ otros servicios $u$ operaciones similares entre transportistas. En la normativa se regulan las modalidades del contrato de viaje, el contenido del contrato mercantil, la forma, el precio, régimen de responsabilidad del organizador del viaje, modificación del contrato y su resolución.

En Chile según Contardo González (2013) se equipara el contrato de viaje al mandato con representación, por el cual el agente se obliga a nombre, riesgo y en favor del usuario, a contratar determinados servicios finales con los prestadores efectivos de estos. De cierta manera esta concepción del contrato de viaje combinado resulta confusa según lo establecido en el artículo 2158 del Código Civil chileno porque la obligación del mandatario consiste en una obligación de medios y de este modo el agente no estaría obligado a que el viaje se realice, sino a emplear todo lo que esté a su alcance para que este se realice. De modo que no podrá sostenerse al mandante de dispensarse de cumplir estas obligaciones a causa de la alegación de que el negocio encomendado al mandatario no fue efectivo, o que pudo desempeñarse a menos costo; salvo que le pruebe culpa.

En Ecuador, el citado Reglamento de Actividades Turísticas considera al contrato de viaje un contrato de turismo ${ }^{7}$, de hecho, se reconoce la modalidad de viajes combinado al incluir un conjunto de servicios previamente programados y ofertados al público por un precio global, o proyectados a solicitud del cliente. Además se hace referencia al momento del perfeccionamiento del contrato, a la necesidad de que el precio, las condiciones y los servicios por escrito y a la posibilidad de la variación del precio previamente justificado.

\section{Manifestación del contrato de viajes en la actividad de intermediación turística cubana}

El contrato típico y en el cual se basa la actividad de intermediación de la A.V. es el contrato de viajes, a través del mismo, el agente se convierte en un proveedor de servicios en una relación de consumo. En correspondencia la A.V. cumple su función principal de mediar entre los prestatarios del servicio turístico con los que anteriormente ha contratado una prestación y los turistas que se convierten en sus clientes.

Por otro lado se encuentran los derechos y obligaciones que se generan de la comercialización del producto turístico que tienen su fuente en el contrato de viajes que suele concertarse entre una A.V. y los consumidores del producto que estas comercializan (González Cabrera, 2012).

En Cuba el contrato de viaje no se halla tipificado en el ordenamiento jurídico, por lo tanto se trata entonces de una figura atípica. Ni el Código de Comercio cubano derogado en materia de contratos, ni el Código Civil han

\footnotetext{
${ }^{7}$ Cfr. Art. 33, Capítulo III del Reglamento general de actividades turísticas publicado en el Registro Oficial nro. 726 del 17 de diciembre del 2002. Contrato de Turismo: en virtud del contrato de turismo la agencia de viajes se obliga para con el cliente, usuario o consumidor a proporcionar los servicios pactados o encomendados mediante el respectivo pago.
} 
regulado el contrato de viaje, ni su forma genérica de viajes combinados; asimismo no lo hace la más reciente regulación cubana de contratación económica el Decreto Ley 304 de 2012, ni el Decreto 310 de los tipos de contrato de igual año.

No obstante, se encuentra una referencia del contrato de viaje combinado en el capítulo primero de la citada Resolución Conjunta 1 de 1999 Reglamento de las AA.VV. cuando se alude al concepto de "paquete turístico". En este apartado el legislador hace mención a la figura contractual al definir el paquete turístico como el viaje en el que se vende por un precio global un conjunto de servicios, que comprenden generalmente el transporte, el alojamiento y otros servicios complementarios (Resolución, 1999).

Sin embargo, no existe en el país otras referencias en la legislación nacional a esta modalidad de contratos de consumo y se ha intentado, por parte de los operadores turísticos, cubrir este espacio con la aplicación de la Convención Internacional del Contrato de Viajes de 1970 y esto se debe a que la T.O. cubana es fundamentalmente realizada por empresas extranjeras con apego a dicha norma de carácter universal (Convención, 1970).

El Convenio de Bruselas no define el contrato de viaje o turismo. Para este el contrato de viaje es una expresión genérica comprensiva de dos especies: a) contrato de organización de viaje, y b) contrato deintermediación de viaje. Asimismo lo diferencia del contrato de transporte y en la misma se distinguen los sujetos que consumen el producto de las AA.VV. en viajero y turista; al mismo tiempo, se define al intermediario de viaje como la persona que puede actuar como un agente de viajes o como un típico mandatario en caso de estar autorizado para actuar y concluir contratos en nombre del prestador del servicio ${ }^{8}$.

En Cuba, los contratos de viajes que celebran las AA.VV. corresponden a la segunda modalidad, aunque por lo general no existen limitaciones para que se centren en un servicio aislado. En correspondencia con el contrato de organización por el cual una persona (organizador de viaje) se obliga, en su propio nombre, mediante un precio global, a prestar un conjunto de servicios combinados de transporte, alojamientos independientes del transporte, u otros servicios u operaciones similares entre transportistas ${ }^{9}$.

De la misma forma, para los agentes cubanos se considera un contrato de consumo aquel en el que se acuerda la prestación de un servicio turístico; y en el mismo interviene la A.V. que intermedia entre el consumidor y quienes realizan la prestación del servicio turístico ${ }^{10}$. En correspondencia la modalidad

\footnotetext{
${ }^{8}$ En el art. 1, inc. 7 se define al viajero como toda persona que se beneficie del compromiso contemplado en el punto 2 y 3 , ya sea que el contrato haya sido concluido o el precio haya sido pagado por ella o por alguien en su nombre. el art. 1, inc. B), de la Convención sobre facultades aduaneras sobre el turismo, define al turista como: toda persona, sin distinción de raza, sexo, lengua o religión, que entre en el territorio de un Estado contratante de aquel en que dicha persona tiene su residencia habitual y permanezca en él 24 horas cuando menos y no más de 6 meses, en cualquier periodo de 12 meses, con fines de turismo, recreo, deportes, salud, asuntos familiares, estudio, peregrinaciones religiosas o negocios sin propósito de inmigración.

${ }^{9}$ Cfr. Artículo 1.2 de la Convención Internacional de Viajes de 1970.

${ }^{10}$ Vid. Artículo 1.3 de la Convención de Bruselas donde se dispone que el contrato de intermediación de viaje es aquel por el cual una persona (agencia de turismo) asume habitualmente la obligación de procurar a otra (viajero o turista), que paga un precio, un contrato de organización de un viaje, o una o algunas de las prestaciones independientes que permitan efectuar un viaje o una estadía cualquiera.
} 
combinada es la que reúne dos o más prestaciones, que entraña la combinación de dos elementos: el transporte y el alojamiento, y otros servicios no directamente reconocidos (Martínez Espín, 1999).

En este contrato turístico las AA.VV. son intermediarias en la prestación a terceros de sus servicios de transporte, hostelería y otros, en ocasión de un viaje o estancia, y de organizadores de viajes resultados de la combinación de aquellos servicios. En ella están presentes dos modalidades básicas: servicios sueltos cuando se facilitan a comisión los elementos aislados de un viaje o estancia; y la de paquetes turísticos que incluye un conjunto de al menos dos prestaciones de servicios previamente programados por la A.V. (alojamiento, transporte y otros).

El perfeccionamiento del contrato de viajes está determinado por la entrega del documento que acredita el servicio contratado: boleto, bono, boucher, entre otros, y a la necesidad de que el precio, las condiciones y los servicios consten por escrito y que su variación dependa de sucesos que previamente se justifiquen y acuerden entre las partes contratantes. En el caso particular de la variación del precio del paquete turístico, es necesario que se establezca una fecha límite previa al viaje, porque sería nulo cualquier cambio en perjuicio del turista en cuanto a los cambios de los valores previamente acordados ${ }^{11}$.

Sin lugar a dudas, el tratamiento de la responsabilidad de los organizadores y detallistas de viajes combinados en cuanto a los daños sufridos por el consumidor, como consecuencia de la no ejecución o ejecución deficiente del contrato, es lo que más debate genera por el nivel de participación de dichas AA.VV. En este caso, la agencia ofrecerá la posibilidad de optar por el reembolso total de lo abonado o su sustitución por otro servicio de características similares en cuanto a categoría y calidad, aunque si con esta sustitución el servicio resulta de inferior categoría o calidad, se debe en su caso, reembolsar la diferencia.

Además, se establece que no se prohíbe revisar el precio, pero se ve con carácter restrictivo esta modificación; lo que garantiza la responsabilidad del organizador del viaje respecto al incumplimiento que pueda producirse respecto de este.

En esta especie del contrato de viajes en el sector distributivo o comercial que lo regula se han desarrollado una serie de determinantes con relación al concepto de organizador en su modalidad no ocasional. Estos se expresan de forma exclusiva en el objeto propio del régimen de las AA.VV. como única empresa cubana de intermediación turística con vocación para realizar este empeño. (Martínez Montenegro, 2015).

A partir de estas particularidades, se deben tener en cuenta para efectuar una mejor contratación del servicio turístico los siguientes elementos:

- Desde el punto de vista económico este contrato exige como requisito sustancial que se realice el viaje por más de veinticuatro horas.

- El hecho de que se efectúe facturación por separado no desvirtúa la naturaleza del viaje combinado, si hay una oferta previa que incluya estas dos prestaciones o elementos, es decir, transporte y alojamiento.

\footnotetext{
${ }^{11} \mathrm{Cfr}$. El artículo 11 de la Convención de Bruselas se dispone que el organizador no puede obtener aumentos del precio global si no es por variaciones en el cambio de moneda o en las tarifas del transportador, y con la condición de que esta facultad haya sido prevista en el documento de viaje. Si el aumento del precio global excede el $10 \%$, el viajero puede rescindir el contrato sin indemnización a su cargo y tiene derecho a que se le reembolse todas las sumas que pagó al organizador de viajes.
} 
- Para que se cumpla el tipo contractual la combinación es de servicios principales, no accesorios ${ }^{12}$.

- Un consumidor puede exigir como requisito previo la organización de las diversas prestaciones, que es lo que se denomina paquete turístico y la asunción jurídica de este concepto es lo que se reconoce como oferta previa. Igualmente, se apuntan de la revisión del módulo de contratación de la A.V. CUBANACAN Varadero, algunos aspectos a tener en cuenta en la concertación del contrato de viajes, por sus propias características y a causa de la especial relación que presentan las AA.VV. y sus clientes:

a) Es irrelevante la finalidad del viaje.

b) Se pretende el mínimo de litigio posible.

c) Si se tiene en cuenta que hay un debilitamiento del turista, quizás emocional o espiritualmente porque se encuentra fuera de su domicilio, de su entorno, hay que suministrar con carácter previo las condiciones del viaje y ello debe aparecer en la oferta ${ }^{13}$.

d) No debe confundirse oferta precontractual con publicidad, porque de lo que se trata es que esta oferta esté vinculada a la parte que la realiza. Asimismo al turista le corresponde recibir información suficiente y veraz sobre las condiciones y características del servicio que desea contratar, a fin de que pueda dar su consentimiento ${ }^{14}$.

e) El contrato debe constar por escrito (forma escrita) y esta formalidad constituye garantía para el consumidor. En tal sentido, hay que entregar copia del contrato al consumidor y lo que no esté contenido expresamente remite a las normas generales de protección y defensa de los consumidores según lo dispuesto en los artículos 15 y 16 de la Convención, hoy en Cuba sin regulación.

f) Puede estipularse contractualmente la posibilidad de la modificación del precio por acuerdo expreso de las partes, y así posibilitar su variación, previa revisión, y en dependencia de esto ajustar los valores del transporte, las tasas de compras y de los servicios.

g) El contrato puede ser modificado por el organizador del viaje, siempre que la modificación introducida no afecte los elementos esenciales del negocio jurídico concertado.

h) Reconocimiento que debe hacerse al turista de su derecho a ser indemnizado económicamente por los daños y perjuicios ocasionados por el incumplimiento o cumplimiento defectuoso de las prestaciones turísticas contratadas. Para ello no solo se deben establecer las alternativas de garantía que establecen en la citada reglamentación cubana, sino un procedimiento operativo que las haga efectivas oportunamente al momento en que el turista deba ser indemnizado ${ }^{15}$.

\footnotetext{
${ }^{12}$ Ejemplo: el dar comida en un avión no entra en la prestación principal de un viaje combinado, esto es accesorio.

${ }^{13}$ En ella, por ejemplo, se debe apuntar si es seguro o no el país a dónde se va, pues funciona como requisito precontractual.

${ }^{14} \mathrm{Cfr}$. Artículo 15 de la Convención Internacional de Viajes establece que II vendedor debe informar a los interesados, por escrito y anticipadamente a la conclusión del contrato, el contenido de las prestaciones debidas relativas al transporte y a la estadía, al precio y a las modalidades de pago, las condiciones de anulación del contrato, y las condiciones para transponer las fronteras.

${ }^{15}$ En la actualidad las AA.VV. cubanas brindan un servicio adicional u overbooking como fórmula para enmendar el servicio afectado. Esta cuestión es discutible en gran medida para el turista internacional que se planifica sus vacaciones en fechas limitadas por un
} 
La mayoría de las condiciones generales se constituyen a razón de la especial relación que existe entre las AA.VV. que organizan los viajes o estadías a sus consumidores y para ello deben poner a disposición de estos últimos, un programa o folleto informativo que señale la oferta sobre el viaje a contratar donde se especifique los servicios y actividades incluidas y sus características. En definitiva este deber de informar es una obligación de la AA.VV., respecto al cliente, de informar los datos, antecedentes o indicaciones sobre el referido bien o servicio ofrecido, en este caso el paquete turístico o las características y condiciones del contrato de viaje combinado, con la finalidad de que el acto de consumo responda efectivamente a las expectativas del consumidor.

En los contratos de viajes se establecen cargas cuyo incumplimiento genera la pérdida de los derechos del viajero, como por ejemplo en los supuestos siguientes: si el usuario no comprueba el horario de salida e itinerario en su A.V. con una determinada antelación al día de la salida previsto, el intermediario queda exonerado de toda responsabilidad; el recibo del equipaje sin protesta escrita del usuario implica que este pierde todo derecho a reclamar; para no perder el derecho al viaje, el pasajero debe presentarse en las oficinas del transportista a la hora señalada con el billete, o en su defecto, con la antelación suficiente que le permita cumplimentar los trámites de salida, la presentación oportuna al embarque es la condición ineludible para solicitar las indemnizaciones en los casos de sobreventa de plazas.

En correspondencia deben existir cláusulas que exoneren la responsabilidad de la agencia por daños en el equipaje, por ser esta más una obligación del transportista y no de la A.V. y el transporte es una de las prestaciones que esta ofrece para el cumplimiento de su finalidad contractual.

\section{CONCLUSIONES}

Los agentes de viajes cubanos establecen sus relaciones económicas a través del contrato económico que convienen con los terceros que prestan el servicio que ellos intermedian, sin embargo, la fuente directa de los derechos y obligaciones que se generan de la comercialización del producto turístico se halla en el contrato de viajes. Este último, es un contrato de consumo que entraña la combinación de dos elementos esenciales: el transporte y el alojamiento, aunque mediante el mismo se pueden vender otros servicios no directamente reconocidos por las AA.VV.

En el contrato de viajes combinado en Cuba, confluyen un conjunto de relaciones de varios servicios turísticos en los cuales se combinan distintos elementos que corresponden a figuras contractuales diferentes, que se fusionan para integrar, en un solo paquete turístico, los intereses del cliente. De este modo, se les reconoce como contratos mixtos con naturaleza mercantil al ser en el plano subjetivo ejecutados generalmente, por empresarios mercantiles, que su actividad turística se desarrolla a través de una actividad económica constitutiva de empresa, la cual se realiza con fines comerciales y de lucro.

período de tiempo. Además este servicio adicional se utiliza para los supuestos de sobre reserva, es decir, cuando el agente vende un número de plazas superiores a las disponibilidades en una aeronave, de un establecimiento hotelero, etc., previendo de antemano que no todos los que reservan su viaje vayan a realizarlo efectivamente. También lo han catalogado como un caso de fuerza mayor, sin embargo, no coincidimos con esta posición ya que son situaciones que atañen a la vinculación contractual del prestador con la agencia que contrata, en efecto, el organizador de viajes está obligado contractualmente a proporcionar transporte y alojamiento en las condiciones pactadas, sin convertirse en una diligencia suficiente de su parte. 


\section{REFERENCIAS BIBLIOGRÁFICAS}

Bech Serrat, J. M. (2007). La responsabilidad contractual de los organizadores y detallistas de viajes combinados. (Tesis doctoral), Universidad de Girona, Girona. Disponible en: http://www.tdx.cat/bitstream/handle/10803/7678/tjmbs.pdf?sequence $=1$.

Cairós Barreto, D. M. (2001). Contrato de mediación laboral y contrato de agencia mercantil: un estudio sobre el objeto del contrato de trabajo. (Tesis doctoral), Universidad de Barcelona, Barcelona. Disponible en: ftp://tesis.bbtk.ull.es/ccssyhum/cs88.pdf

Contardo González, J. (2012). La responsabilidad de las AA.VV. por overbooking o exceso de reservas. En la Semana Jurídica 29. Santiago: Legal publishing, Thomson Reuters.

Convención Internacional de Contratos de Viajes (CIV), suscripta en Bruselas, el 23 de abril de 1970.

Decreto Ley 304 y Decreto 310 de fecha 1 de noviembre de 2012 publicado en Gaceta Oficial No 62, La Habana.

Directiva 90/314 de la Comunidad de Estados Europeos del Consejo de las Comunidades Europeas de 1992, que adecua Convenio de Bruselas del 23 de abril de 1970 relativo al contrato internacional de viajes combinados.

García Rubio, M. P. (1999). La responsabilidad contractual de las agencias de viaje (Montecorvo Ed.). España: Madrid.

Gómez Calero, J. (1975). Sobre la mercantilidad de las cooperativas. Revista de Derecho Mercantil, 137, 301-328.

Guevara Hernández, E. (2009). Procedimiento para los análisis del producto opcionales en la agencia de viajes Gaviota Tours Varadero. (Tesis maestría), Universidad de Matanzas "Camilo Cienfuegos", Matanzas.

Kemelmajer Carlucci, A. (2006). El contrato de servicios turísticos. Revista Latinoamericana de Derecho, 5, 97-128. Disponible en:

http://info.juridicas.unam.mx/publica/librev/rev/revlad/cont/5/cnt/cnt6.pdf.

Ley 21/ 1995 de viajes combinados en España.

Ley núm. 59 Código Civil cubano de fecha 16 de julio de 1987.

Martínez Espín, P. (1999). El contrato de viaje combinado (Antecedentes, Derecho Comparado, Estudio Normativo y Jurisprudencial), Ed. Universidad de Castilla-La Mancha, Cuenca.

Martínez Montenegro, I. (2012). Responsabilidad contractual de las agencias de viajes receptivas. Revista Retos Turísticos, 2, 124-132. Disponible en: retos.mes.edu.cu/index.php/retojs/article/view/143

----.-. (2014). Procedimiento para el análisis del marco legal regulatorio de las agencia de viajes receptivas de Varadero. Caso Cubanacán S.A. (Tesis de maestría), Universidad de Matanzas "Camilo Cienfuegos", Matanzas.

------.-. (2015). Realidades del contrato de viajes combinado en Cuba. En L. Pérez (Presidencia), XV Jornadas Internacionales de Derecho de Contratos. Simposio dirigido por la Unión Nacional de Juristas de Cuba y la Facultad de Derecho de la Universidad de La Habana, La Habana.

Reglamento de agencias de viajes nacionales, sucursales y representaciones de agencias de viajes extranjeras en la República de Cuba § Resolución Conjunta No. 1 (1998).http://www.camaracuba.cu/index.php/es/.../30-registros?...resolucion-11998.

Sanz Domínguez, C. (2005). Régimen jurídico-administrativo de las Empresas de Intermediación turísticas. (Tesis doctoral), Universidad de Sevilla, Sevilla. Disponible en:

http://www.us.es/downloads/estudios/master/listado_profesorado_due.pdf

Valero Jiménez, B., Suárez Mella, R. (2010). Modelo de medición de excelencia empresarial en agencias de viajes. Revista Retos Turísticos. Disponible en: retos.mes.edu.cu/index.php/retojs/article/viewFile/88/78 